\title{
Condensate formation with three-component ultracold fermions
}

\author{
Luca Salasnich \\ INO-CNR, Dipartimento di Fisica "Galileo Galilei", \\ Università di Padova, Via Marzolo 8, 35131 Padova, Italy
}

(Dated: November 15, 2018)

\begin{abstract}
We investigate the formation of Bose-Einstein condensation and population imbalance in a threecomponent Fermi superfluid by increasing the U(3) invariant attractive interaction. We consider the system at zero temperature in three dimensions and also in two dimensions. Within the meanfield theory, we derive explicit formulas for number densities, gap order parameter, condensate density and condensate fraction of the uniform system, and analyze them in the crossover from the Bardeen-Cooper-Schrieffer (BCS) state of Cooper pairs to the Bose-Einstein Condensate (BEC) of strongly-bound molecular dimers. In addition, we study this Fermi mixture trapped by a harmonic potential: we calculate the density profiles of the three components and the condensate density profile of Cooper pairs in the BCS-BEC crossover.
\end{abstract}

PACS numbers: 03.75.Hh, 03.75.Ss

\section{INTRODUCTION}

In the last years two experimental groups [1, 2] have analyzed the condensate fraction of ultra-cold two hyperfine component Fermi vapors of ${ }^{6} \mathrm{Li}$ atoms in the crossover from the Bardeen-Cooper-Schrieffer (BCS) state of Cooper Fermi pairs to the Bose-Einstein condensate (BEC) of molecular dimers. These experiments are in quite good agreement with mean-field theoretical predictions [3, 4] and Monte-Carlo simulations [5] at zero temperature, while at finite temperature beyond-meanfield corrections are needed [6].

Degenerate three-component gases have been experimentally realized using the three lowest hyperfine states of ${ }^{6} \mathrm{Li}$ [7, 8]. At high magnetic fields the scattering lengths of this three-component system are very close each other and the system is approximately $\mathrm{U}(3)$ invariant. Moreover, it has been theoretically predicted that good $S U(N)$ invariance (with $N \leq 10$ ) can be reached with ultracold alkaline-earth atoms (e.g. with ${ }^{87} \mathrm{Sr}$ atoms) 9]. Very recently, Ozawa and Baym have investigated the BCS-BEC crossover of uniform threecomponent ultracold fermions with $\mathrm{U}(3)$ symmetry at zero and finite temperature in three-dimensional space [10].

In this paper we focus on the condensate formation in a uniform three-component Fermi superfluid by increasing the $\mathrm{U}(3)$ invariant attractive interaction. We determine the condensate fraction and the population imbalance of the system in both the three-dimensional case and in the two-dimensional one. By using the extended BEC theory [11], we obtain analytical formulas for number densities, condensate density and condensate fraction [3, 12] of the uniform system and analyze them in the crossover from the Bardeen-Cooper-Schrieffer (BCS) state of Cooper pairs to the Bose-Einstein Condensate (BEC) of strongly-bound molecular dimers. We consider also the inclusion of a harmonic confinement, determining the density profiles of the three components of the ultracold gas, and the space dependence of condensate density and gap order parameter. In the paper we neglect effects of three-body interactions like Efimov three-body bound states.

\section{THE MODEL}

The shifted Hamiltonian density of a dilute and interacting three-component Fermi gas in a volume $V$ is given by [10]

$$
\begin{aligned}
\hat{\mathcal{H}}^{\prime} & =\sum_{\alpha=R, G, B} \hat{\psi}_{\alpha}^{+}\left(-\frac{\hbar^{2}}{2 m} \nabla^{2}-\mu\right) \hat{\psi}_{\alpha}+g\left(\hat{\psi}_{R}^{+} \hat{\psi}_{G}^{+} \hat{\psi}_{G} \hat{\psi}_{R}\right. \\
& \left.+\hat{\psi}_{R}^{+} \hat{\psi}_{B}^{+} \hat{\psi}_{B} \hat{\psi}_{R}+\hat{\psi}_{G}^{+} \hat{\psi}_{B}^{+} \hat{\psi}_{B} \hat{\psi}_{G}\right)
\end{aligned}
$$

where $\hat{\psi}_{\alpha}(\mathbf{r})$ is the field operator that destroys a fermion of component $\alpha$ in the position $\mathbf{r}$, while $\hat{\psi}_{\alpha}^{+}(\mathbf{r})$ creates a fermion of component $\alpha$ in $\mathbf{r}$. To mimic QCD the three components are thought as three colors: red $(\mathrm{R})$, green $(\mathrm{G})$ and blue $(\mathrm{B})$. The attractive inter-atomic interaction is described by a contact pseudo-potential of strength $g$ $(g<0)$. The number density operator is

$$
\hat{n}(\mathbf{r})=\sum_{\alpha=R, G, B} \hat{\psi}_{\alpha}^{+}(\mathbf{r}) \hat{\psi}_{\alpha}(\mathbf{r})
$$

and the average number of fermions reads

$$
N=\int d^{3} \mathbf{r}\langle\hat{n}(\mathbf{r})\rangle .
$$

This total number $N$ is fixed by the chemical potential $\mu$ which appears in Eq. (11). As stressed in Ref. [10], by fixing only the total chemical potential $\mu$ (or equivalently only the total number of atoms $N$ ) the Hamiltonian (1) is invariant under global $\mathrm{U}(3)$ rotations of the species (and consequently also under global SU(3)). At zero temperature, the attractive interaction leads to pairing of fermions which breaks the U(3) symmetry but only two colors are paired and one is left unpaired [10]. 
Following Ozawa and Baym [10], we assume without loss of generality that the red and green particles are paired and the blue are not paired. The interacting terms can be then treated within the minimal mean-field BCS approximation, giving

$$
g \hat{\psi}_{R}^{+} \hat{\psi}_{G}^{+} \hat{\psi}_{G} \hat{\psi}_{R}=g\left\langle\hat{\psi}_{R}^{+} \hat{\psi}_{G}^{+}\right\rangle \hat{\psi}_{G} \hat{\psi}_{R}+g \hat{\psi}_{R}^{+} \hat{\psi}_{G}^{+}\left\langle\hat{\psi}_{G} \hat{\psi}_{R}\right\rangle
$$

and

$$
g \hat{\psi}_{R}^{+} \hat{\psi}_{B}^{+} \hat{\psi}_{B} \hat{\psi}_{R}=g \hat{\psi}_{G}^{+} \hat{\psi}_{B}^{+} \hat{\psi}_{B} \hat{\psi}_{G}=0 .
$$

Notice that the Hartree terms have been neglected, while the pairing gap $\Delta=g\left\langle\hat{\psi}_{G} \hat{\psi}_{R}\right\rangle$ between red and green fermions is the key quantity.

The shifted Hamiltonian density (11) is diagonalized by using the Bogoliubov-Valatin representation of the field operator $\hat{\psi}_{\alpha}(\mathbf{r})$ in terms of the anticommuting quasiparticle Bogoliubov operators $\hat{b}_{\mathbf{k} \alpha}$ with amplitudes $u_{k}$ and $v_{k}$ and energy $E_{k}$. After minimization of the resulting quadratic Hamiltonian one finds familiar expressions for these quantities:

$$
E_{k}=\left[\left(\frac{\hbar^{2} k^{2}}{2 m}-\mu\right)^{2}+\Delta^{2}\right]^{1 / 2}
$$

and

$$
v_{k}^{2}=\frac{1}{2}\left(1-\frac{\frac{\hbar^{2} k^{2}}{2 m}-\mu}{E_{k}}\right),
$$

with $u_{k}^{2}=1-v_{k}^{2}$. In addition we find the equation for the number of particles

$$
N=N_{R}+N_{G}+N_{B},
$$

where

$$
N_{R}=N_{G}=\frac{1}{2} \sum_{\mathbf{k}} v_{k}^{2}
$$

and

$$
N_{B}=\sum_{\mathbf{k}} \Theta\left(\mu-\frac{\hbar^{2} k^{2}}{2 m}\right)
$$

with $\Theta(x)$ the Heaviside step function, and also the gap equation

$$
-\frac{1}{g}=\frac{1}{V} \sum_{\mathbf{k}} \frac{1}{2 E_{k}} .
$$

The chemical potential $\mu$ and the gap energy $\Delta$ are obtained by solving equations (8) and (11).

We observe that the number of red-green pairs in the lowest state, i.e. the condensate number of red-green pairs, is given by $[\underline{3}, 4,[6]$

$$
N_{0}=\int d^{3} \mathbf{r}_{1} d^{3} \mathbf{r}_{2}\left|\left\langle\hat{\psi}_{G}\left(\mathbf{r}_{1}\right) \hat{\psi}_{R}\left(\mathbf{r}_{2}\right)\right\rangle\right|^{2},
$$

and it is straightforward to show that

$$
N_{0}=\sum_{\mathbf{k}} u_{k}^{2} v_{k}^{2} .
$$

In the continuum limit, due to the choice of a contact potential, the gap equation (11) diverges in the ultraviolet. This divergence is linear in three dimensions and logarithmic in two dimensions. Let us face this problem in the next two sections.

\section{THREE-COMPONENT ULTRACOLD FERMIONS IN THREE DIMENSIONS}

In three dimensions, a suitable regularization [11] is obtained by introducing the inter-atomic scattering length $a_{F}$ via the equation

$$
-\frac{1}{g}=-\frac{m}{4 \pi \hbar^{2} a_{F}}+\frac{1}{V} \sum_{\mathbf{k}} \frac{m}{\hbar^{2} k^{2}},
$$

and then subtracting this equation from the gap equation (11). In this way one obtains the three-dimensional regularized gap equation

$$
-\frac{m}{4 \pi \hbar^{2} a_{F}}=\frac{1}{V} \sum_{\mathbf{k}}\left(\frac{1}{2 E_{k}}-\frac{m}{\hbar^{2} k^{2}}\right) .
$$

In the three-dimensional continuum limit $\sum_{\mathbf{k}} \rightarrow$ $V /(2 \pi)^{3} \int d^{3} \mathbf{k} \rightarrow V /\left(2 \pi^{2}\right) \int k^{2} d k$ from the number equation (8) with (9) and (10) we find the total number density as

$$
n=\frac{N}{V}=n_{R}+n_{G}+n_{B},
$$

with

$$
n_{R}=n_{G}=\frac{1}{2} \frac{(2 m)^{3 / 2}}{2 \pi^{2} \hbar^{3}} \Delta^{3 / 2} I_{2}\left(\frac{\mu}{\Delta}\right)
$$

and

$$
n_{B}=\frac{1}{3} \frac{(2 m)^{3 / 2}}{2 \pi^{2} \hbar^{3}} \mu^{3 / 2} \Theta(\mu) .
$$

The renormalized gap equation (15) becomes instead

$$
-\frac{1}{a_{F}}=\frac{2(2 m)^{1 / 2}}{\pi \hbar^{3}} \Delta^{1 / 2} I_{1}\left(\frac{\mu}{\Delta}\right),
$$

where $k_{F}=(6 \pi N /(3 V))^{1 / 3}=\left(2 \pi^{2} n\right)^{1 / 3}$ is the Fermi wave number. Here $I_{1}(x)$ and $I_{2}(x)$ are the two monotonic functions

$$
\begin{gathered}
I_{1}(x)=\int_{0}^{+\infty} y^{2}\left(\frac{1}{\sqrt{\left(y^{2}-x\right)^{2}+1}}-\frac{1}{y^{2}}\right) d y, \\
I_{2}(x)=\int_{0}^{+\infty} y^{2}\left(1-\frac{y^{2}-x}{\sqrt{\left(y^{2}-x\right)^{2}+1}}\right) d y,
\end{gathered}
$$


which can be expressed in terms of elliptic integrals, as shown by Marini, Pistolesi and Strinati [11]. In a similar way we get the condensate density of the red-green pair as

$$
n_{0}=\frac{N_{0}}{V}=\frac{m^{3 / 2}}{8 \pi \hbar^{3}} \Delta^{3 / 2} \sqrt{\frac{\mu}{\Delta}+\sqrt{1+\frac{\mu^{2}}{\Delta^{2}}}} .
$$

This equation and the gap equation (19) are the same of the two-component superfluid fermi gas (see [3]) but the number equation (8), with (9) and (10), is clearly different. Note that all the relevant quantities can be expressed in terms of the ratio

$$
x_{0}=\frac{\mu}{\Delta} \text {. }
$$

For instance, the fraction of red fermions, which is equal to the fraction of green fermions, is given by

$$
\frac{n_{R}}{n}=\frac{n_{G}}{n}=\frac{I_{2}\left(x_{0}\right)}{2 I_{2}\left(x_{0}\right)+\frac{2}{3} x_{0}^{3 / 2} \Theta\left(x_{0}\right)},
$$

while the fraction of blue fermions reads

$$
\frac{n_{B}}{n}=1-\frac{I_{2}\left(x_{0}\right)}{I_{2}\left(x_{0}\right)+\frac{1}{3} x_{0}^{3 / 2} \Theta\left(x_{0}\right)} .
$$

The fraction of condensed red-green pairs is instead

$$
\frac{n_{0}}{n}=\frac{\pi}{8 \sqrt{2}} \frac{\sqrt{x_{0}+\sqrt{1+x_{0}^{2}}}}{I_{2}\left(x_{0}\right)+\frac{1}{3} x_{0}^{3 / 2} \Theta\left(x_{0}\right)} .
$$

Finally, the adimensional interaction strength of the BCS-BEC crossover is given by

$$
y=\frac{1}{k_{F} a_{F}}=-\frac{2}{\pi} \frac{I_{1}\left(x_{0}\right)}{2 I_{2}\left(x_{0}\right)+\frac{2}{3} x_{0}^{3 / 2} \Theta\left(x_{0}\right)} .
$$

We can use these parametric formulas of $x_{0}$ to plot the density fractions as a function of the scaled interaction strength $y$.

In the upper panel of Fig. 1 we plot the fraction of red fermions $n_{R} / n$ (solid line) and the fraction of blue fermions $n_{B} / n$ (dashed line) as a function of scaled interaction strength $y=1 /\left(k_{F} a_{F}\right)$. The behavior of $n_{G} / n$ is not shown because it is exactly the same of $n_{R} / n$. The figure shows that in the deep BCS regime $(y \ll-1)$ the system has $n_{R} / n=n_{G} / n=n_{B} / n=1 / 3$. By increasing $y$ the fraction of red and green fermions increases while the fraction of blue fermions decreases. At $y \simeq 0.6$, where $x_{0}=0$, the fraction of blue fermions becomes zero, i.e. $n_{B} / n=0$ and consequently $n_{R} / n=n_{G} / n=1 / 2$. For larger values of $y$ there are only the paired red and green particles. This behavior is fully consistent with the findings of Ozawa and Baym [10].

In the lower panel of Fig [1 it is shown the plot of the condensate fraction $n_{0} /(n / 2)$ of red-green pairs through the BCS-BEC crossover as a function of the Fermi-gas parameter $y=1 /\left(k_{F} a_{F}\right)$. The figure shows that a large condensate fraction builds up in the BCS side already before the unitarity limit $(y=0)$, and that on the BEC side $(y \gg 1)$ it rapidly converges to one.
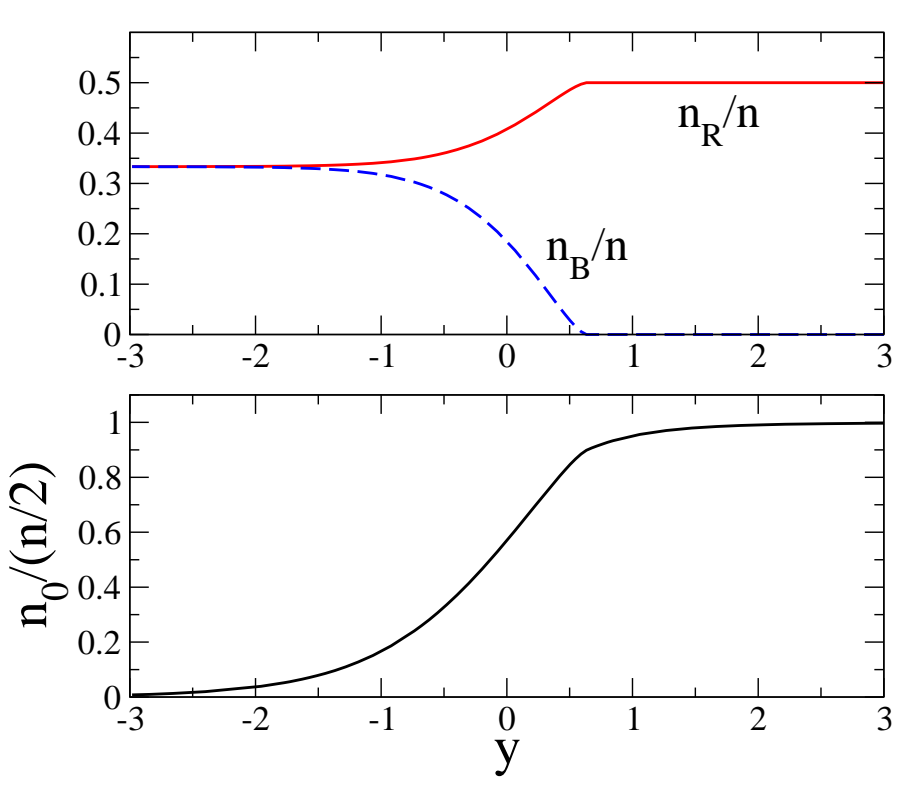

FIG. 1: (Color online). Three-component ultracold fermions in three-dimensions. Upper panel: fraction of red fermions $n_{R} / n$ (solid line) and fraction of blue fermions $n_{B} / n$ (dashed line) as a function of scaled interaction strength $y=$ $1 /\left(k_{F} a_{F}\right)$. Lower panel: condensed fraction of red-green particles $n_{0} / n$ as a function of scaled interaction strength $y=1 /\left(k_{F} a_{F}\right)$.

\section{THREE-COMPONENT ULTRACOLD FERMIONS IN TWO DIMENSIONS}

A two-dimensional Fermi gas can be obtained by imposing a very strong confinement along one of the three spatial directions. In practice, the potential energy $E_{p}$ of this strong external confinement must be much larger than the total chemical potential $\mu_{3 D}$ of the fermionic system: $\mu_{3 D} \ll 2 E_{P}$ [14]. Contrary to the threedimensional case, in two dimensions quite generally a bound-state energy $\epsilon_{B}$ exists for any value of the interaction strength $g$ between atoms [11]. For the contact potential the bound-state equation is

$$
-\frac{1}{g}=\frac{1}{V} \sum_{\mathbf{k}} \frac{1}{\frac{\hbar^{2} k^{2}}{2 m}+\epsilon_{B}}
$$

and then subtracting this equation from the gap equation (11) one obtains the two-dimensional regularized gap equation [1]

$$
\sum_{\mathbf{k}}\left(\frac{1}{\frac{\hbar^{2} k^{2}}{2 m}+\epsilon_{B}}-\frac{1}{2 E_{k}}\right)=0 .
$$

Note that, for a 2D inter-atomic potential described by a $2 \mathrm{D}$ circularly symmetric well of radius $R_{0}$ and depth $U_{0}$, the bound-state energy $\epsilon_{B}$ is given by $\epsilon_{B} \simeq$ $\hbar^{2} /\left(2 m R_{0}^{2}\right) \exp \left(-2 \hbar^{2} /\left(m U_{0} R_{0}^{2}\right)\right)$ with $U_{0} R_{0}^{2} \rightarrow 0$ [13].

In the two-dimensional continuum limit $\sum_{\mathbf{k}} \rightarrow$ 

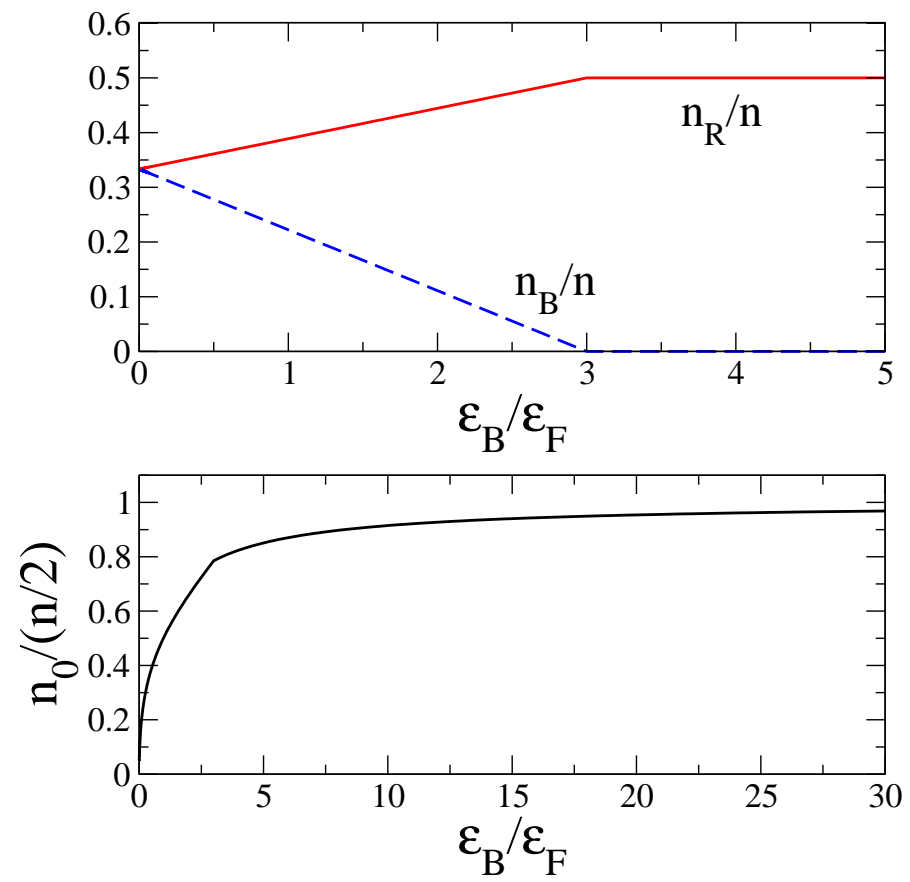

FIG. 2: (Color online). Three-component ultracold fermions in two-dimensions. Upper panel: fraction of red fermions $n_{R} / n$ (solid line) and fraction of blue fermions $n_{B} / n$ (dashed line) as a function of scaled bound-state energy $\epsilon_{B} / \epsilon_{F}$. Lower panel: condensed fraction of red-green particles $n_{0} / n$ as a function of scaled bound-state energy $\epsilon_{B} / \epsilon_{F}$.

$V /(2 \pi)^{2} \int d^{2} \mathbf{k} \rightarrow V /(2 \pi) \int k d k$, the Eq. (29) gives

$$
\epsilon_{B}=\Delta\left(\sqrt{1+\frac{\mu^{2}}{\Delta^{2}}}-\frac{\mu}{\Delta}\right) .
$$

Instead, the number equation (8) with (9) and (10) gives the total number density as

$$
n=\frac{N}{V}=n_{R}+n_{G}+n_{B},
$$

where $V$ is a two-dimensional volume (i.e. an area), the red and green densities are

$$
n_{R}=n_{G}=\frac{1}{2}\left(\frac{m}{2 \pi \hbar^{2}}\right) \Delta\left(\frac{\mu}{\Delta}+\sqrt{1+\frac{\mu^{2}}{\Delta^{2}}}\right),
$$

while the blue density reads

$$
n_{B}=\left(\frac{m}{2 \pi \hbar^{2}}\right) \mu \Theta(\mu) .
$$

Finally, the condensate density of red-green pairs is given by

$$
n_{0}=\frac{1}{4}\left(\frac{m}{2 \pi \hbar^{2}}\right) \Delta\left(\frac{\pi}{2}+\arctan \left(\frac{\mu}{\Delta}\right)\right) .
$$

Also in this two-dimensional case all the relevant quantities can be expressed in terms of the ratio $x_{0}=\mu / \Delta$. In particular, the fraction of red fermions, which is equal to the fraction of green fermions, is given by

$$
\frac{n_{R}}{n}=\frac{n_{G}}{n}=\frac{x_{0}+\sqrt{1+x_{0}^{2}}}{2\left[x_{0}+\sqrt{1+x_{0}^{2}}+x_{0} \Theta\left(x_{0}\right)\right]},
$$

the fraction of blue fermions is

$$
\frac{n_{B}}{n}=1-2 \frac{n_{R}}{n},
$$

and the condensate fraction is

$$
\frac{n_{0}}{n}=\frac{\frac{\pi}{2}+\arctan \left(x_{0}\right)}{4\left[x_{0}+\sqrt{1+x_{0}^{2}}+x_{0} \Theta\left(x_{0}\right)\right]} .
$$

In two-dimensions the Fermi energy $\epsilon_{F}=\hbar^{2} k_{F}^{2} /(2 m)$ of a non-interacting Fermi gas is given by $\epsilon_{F}=\pi \hbar^{2} n / m$ with $k_{F}=(4 \pi N /(3 V))^{1 / 2}=(4 \pi n / 3)^{1 / 2}$ the Fermi wave number. It is convenient to express the bound-state energy $\epsilon_{B}$ in terms of the Fermi energy $\epsilon_{F}$. In this way we find

$$
\frac{\epsilon_{B}}{\epsilon_{F}}=3 \frac{\sqrt{1+x_{0}^{2}}-x_{0}}{x_{0}+\sqrt{1+x_{0}^{2}}+x_{0} \Theta\left(x_{0}\right)},
$$

We can now use these parametric formulas of $x_{0}$ to plot the fractions as a function of the scaled bound-state en$\operatorname{ergy} \epsilon_{B} / \epsilon_{F}$.

In the upper panel of Fig. 2 we plot the fraction of red fermions $n_{R} / n$ (solid line) and the fraction of blue fermions $n_{B} / n$ (dashed line) as a function of scaled bound-state energy $\epsilon_{B} / \epsilon_{F}$. The behavior of $n_{G} / n$ is not shown because it is exactly the same of $n_{R} / n$. The figure shows that in the deep BCS regime $\left(\epsilon_{B} / \epsilon_{F} \ll 1\right)$ the system has $n_{R} / n=n_{G} / n=N_{B} / n=1 / 3$. By increasing $\epsilon_{B} / \epsilon_{F}$ the fraction of red and green fermions increases while the fraction of blue fermions decreases. At $\epsilon_{B} / \epsilon_{F}=3$, where $x_{0}=0$, the fraction of blue fermions becomes zero. For larger values of $\epsilon_{B} / \epsilon_{F}$ there are only the paired red and green particles. This behavior is quite similar to the one of the three-dimensional case; the main difference is due to the fact that here the curves are linear.

In the lower panel of Fig 2 it is shown the condensate fraction $n_{0} /(n / 2)$ of red-green pairs. In the weaklybound BCS regime $\left(\epsilon_{B} / \epsilon_{F} \ll 1\right)$ the condensed fraction $n_{0} / n$ goes to zero, while in the strongly-bound BEC regime $\left(\epsilon_{B} / \epsilon_{F} \gg 1\right)$ the condensed fraction $n_{0} / n$ goes to $1 / 2$, i.e. all the red-green Fermi pairs belong to the Bose-Einstein condensate. Notice that the condensate fraction is zero when the bound-state energy $\epsilon_{B}$ is zero. For small values of $\epsilon_{B} / \epsilon_{F}$ the condensed fraction has a very fast grow but then it reaches the asymptotic value $1 / 2$ very slowly. 

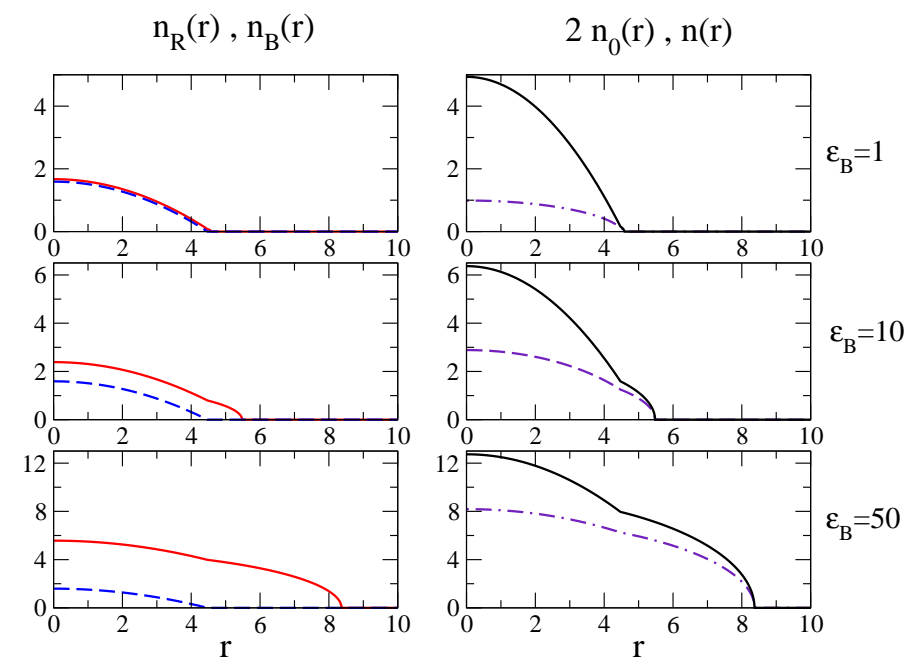

FIG. 3: (Color online). Three-component ultracold fermions in two-dimensions under harmonic confinement $U(r)=r^{2} / 2$. Left panels: density profile $n_{R}(r)$ of red fermions (solid lines) and density profile $n_{B}(r)$ of blue fermions (dashed lines). Right panels: total density profile $n(r)=2 n_{R}(r)+n_{B}(r)$ (solid lines) and condensate density profile $2 n_{0}(r)$ (dot-dashed lines). Note that the density $n_{G}(r)$ of green fermions is not shown because it is equal to $n_{R}(r)$. Data obtained with $\bar{\mu}=10$ and three values of the bound-state energy $\epsilon_{B}: \epsilon_{B}=1$ (upper panels), $\epsilon_{B}=10$ (middle panels), and $\epsilon_{B}=50$ (lower panels). We use scaled variables where lengths are in units of $a_{H}=\sqrt{\hbar /(m \omega)}$, with $\omega$ the frequency of harmonic confinement, and densities in units of $a_{H}^{-2}$.

\section{INCLUSION OF HARMONIC CONFINEMENT}

It is interesting to study the effect of a harmonic potential

$$
U(r)=\frac{1}{2} m \omega^{2} r^{2}
$$

on the properties of the three-component ultracold gas in the BCS-BEC crossover. For semplicity we investigate the two-dimensional case, which gives rise to elegant formulas also in this non-uniform configurtion. In fact, by using the local density approximation, namely the substitution

$$
\mu \rightarrow \mu(r)=\bar{\mu}-U(r),
$$

the gap equation (30) gives the space-dependent gap parameter as

$$
\Delta(r)=\Delta_{0}\left(1-\frac{r^{2}}{r_{0}^{2}}\right) \Theta\left(1-\frac{r^{2}}{r_{0}^{2}}\right),
$$

where $\Delta_{0}=\sqrt{\epsilon_{B}^{2}+2 \epsilon_{B} \bar{\mu}}$ and $r_{0}=\Delta_{0} / \sqrt{\epsilon_{B} m \omega}$. Here $\bar{\mu}$ is the chemical potential of the non-uniform system.

In the same way the density profiles of red, green and blue fermions read

$$
\begin{gathered}
n_{R}(r)=n_{G}(r)=\frac{1}{2}\left(\frac{m}{2 \pi \hbar^{2}}\right) \Delta(r)\left(\frac{\mu(r)}{\Delta(r)}+\sqrt{1+\frac{\mu(r)^{2}}{\Delta(r)^{2}}}\right), \\
n_{B}(r)=\left(\frac{m}{2 \pi \hbar^{2}}\right) \mu(r) \Theta(\mu(r)) .
\end{gathered}
$$

The density profile of condensed red-geen pairs is instead given by

$$
n_{0}(r)=\frac{1}{4}\left(\frac{m}{2 \pi \hbar^{2}}\right) \Delta(r)\left(\frac{\pi}{2}+\arctan \left(\frac{\mu(r)}{\Delta(r)}\right)\right) .
$$

In Fig. 3 we plot results obtained by using scaled variables: energy in units of $\hbar \omega$ and length in units of $a_{H}=\sqrt{\hbar /(m \omega)}$. We work at fixed chemical potential $\bar{\mu}=10$ and increase the bound-state energy $\epsilon_{B}$. In the upper panels of Fig. 3 we set $\epsilon_{B}=1$, in the middle panels $\epsilon_{B}=10$ and in the lower ones $\epsilon_{B}=50$. In the left panels of Fig. 3 we show density profiles of red particles (solid lines) and blue particles (dashed lines). In the right panels we show instead the total density profile $n(r)=n_{R}(r)+n_{G}(r)+n_{B}(r)$ (solid line) and the density profile $2 n_{0}(r)$ of condensed particles (dot-dashed lines). As expected, by increasing $\epsilon_{B}$ the critical radius $r_{0}=\sqrt{\epsilon_{B}+2 \bar{\mu}}$ (written in scaled units), at which the gap order parameter $\Delta(r)$ and the condensate density $n_{0}(r)$ go to zero, becomes much larger than the critical radius $r_{T}=\sqrt{2 \bar{\mu}}$, at which the density $n_{B}(r)$ of blue fermions goes to zero. Moreover, by increasing $\epsilon_{B}$ the cloud of blue particles becomes quite small while the cloud of condensed particles approaches the total one.

\section{CONCLUSIONS}

We have investigated the condensate fraction and the population imbalance of a uniform three-component ultracold fermions by increasing the $\mathrm{U}(3)$ invariant attractive interaction. We have considered the superfluid system both in the three-dimensional case and in the twodimensional one. For the uniform system we have obtained explicit formulas and plots for number densities, condensate density and population imbalance in the full BCS-BEC crossover. For the system under harmonic confinement we have analyzed the density profiles of the three components and the density profile of the condensed pairs by varying the interaction strength. In our calculations we have used the mean-field extended BCS theory. Monte Carlo simulations have shown that, at zero-temperature, beyond-mean-field effects are negligible in the BCS side of the BCS-BEC crossover while they become relevant in the BEC side [5, 15]. Nevertheless, in the deep BEC side the condensate fraction goes in any case to one and the main difference in its determination is around the unitarity limit, where the relative difference between mean-field and Monte Carlo condensate fraction 
is about $20 \%$ [5]. We think that our results can be of interest for next future experiments with degenerate gases made of alkali-metal or alkaline-earth atoms. As stressed in the introduction, $\mathrm{SU}(\mathrm{N})$ invariant interactions can be experimentaly obtained by using these atomic species [79]. The problem of unequal couplings, and also that of a fixed number of atoms for each component, is clearly of big interest too, and its analysis can be afforded by including more than one order parameter (see for instance [16]).

The author thanks Flavio Toigo for useful discussions and Nicola Manini for computational help.
[1] M.W. Zwierlein et al., Phys. Rev. Lett. 92, 120403 (2004); M.W. Zwierlein, C.H. Schunck, C.A. Stan, S.M.F. Raupach, and W. Ketterle, Phys. Rev. Lett. 94, 180401 (2005).

[2] Y. Inada, M. Horikoshi, S. Nakajima, M. KuwataGonokami, M. Ueda, and T. Makaiyama, Phys. Rev. Lett. 101, 180406 (2008).

[3] L. Salasnich, N. Manini, and A. Parola, Phys. Rev. A 72, 023621 (2005).

[4] G. Ortiz and J. Dukelsky, Phys. Rev. A 72, 043611 (2005).

[5] G. E. Astrakharchik, J. Boronat, J. Casulleras, and S. Giorgini, Phys. Rev. Lett. 95, 230405 (2005).

[6] Y. Ohashi and A. Griffin, Phys. Rev. A 72, 063606 (2005); N. Fukushima, Y. Ohashi, E. Taylor, and A. Griffin, Phys. Rev. A 75, 033609 (2007).

[7] T.B. Ottenstein, T. Lompe, M. Kohen, A.N. Wenz, and S. Jochim, Phys. Rev. Lett. 101, 203202 (2008).
[8] J.H. Huckans, J.R. Williams, E.L. Hazlett, R.W. Sites, and K.M. OfHara, Phys. Rev. Lett. 102, 165302 (2009).

[9] A.V. Gorshkov, M. Hermele, V. Gurarie, C. Xu, P.S. Julienne, J. Ye, P. Zoller, E. Demler, M.D. Lukin, A.M. Rey, Nature Physics 6, 289 (2010).

[10] T. Ozawa and G. Baym, Phys. Rev. A 82, 063615 (2010).

[11] M. Marini, F. Pistolesi, and G.C. Strinati, Eur. Phys. J. B 1, 151 (1998).

[12] L. Salasnich, Phys. Rev. A 76, 015601 (2007).

[13] L.D. Landau and E.M. Lifshitz, Quantum Mechanics. Non Relativistic Theory. Course of Theoretical Physics, Vol. 3 (Pergamon Press, New York, 1989).

[14] G. Mazzarella, L. Salasnich, and F. Toigo, Phys. Rev. A 79, 023615 (2009).

[15] G. Bertaina and S. Giorgini, arXiv:1011.3737.

[16] O.H.T. Nummi, J.J. Kinnunen, and P. Torma, arXiv:1011.5336 